\title{
Mitochondria isolated from the striatum brain of acute paraquat treated rats exhibit a higher degree of oxidative phosphorylation coupling, which shows that they are not subject to energetic dysfunction upon acute paraquat administration
}

\author{
D. A. Rendon ${ }^{1}$
}

Received: 25 June 2016 / Accepted: 9 August 2016 / Published online: 29 September 2016

(C) Springer Science+Business Media New York 2016

In the recent article by Czerniczyniec et al. (2015) entitled "Impairment of striatal mitochondrial function by acute paraquat poisoning", published in the journal J Bioenerg Biomembr 2015; 47(5): 395-408, various aspects of the bioenergetics of mitochondria isolated from the striatum and the cerebral cortex of the brain during acute paraquat toxicity have been investigated. The bioenergetic aspects studied in this investigation are basically fall into two large categories: i) the determination of the state 4 mitochondrial respiration (state 4), the state 3 mitochondrial respiration (state 3 ), and the respiratory control ratio (RC); and ii) the determination of the activity of mitochondrial complex I-III, mitochondrial complex II-III, and mitochondrial complex IV. On the basis of all of these results, Czerniczyniec et al. (2015) conclude the existence of a paraquat dysfunction in the bioenergetics of mitochondria isolated from the striatum of the brain. However, both a part of the methodology used and the interpretation of the above-mentioned results obtained exhibit serious fallacies and contradictions that do not support the conclusion of the authors with respect to a negative alteration of the bioenergetics of the striatum mitochondria upon acute paraquat administration.

The data of Fig. 2a-KCN-sensitive respiration (in the article by Czerniczyniec et al. 2015) correspond to the consumption

This comment refers to the article available at doi:10.1007/s10863-0159624-x.

An author's reply to this comment is available at doi:10.1007/s10863016-9680-x.

D. A. Rendon

darendon@unal.edu.co

1 Facultad de Ciencias - Escuela de Física - Laboratorio de Biofísica, Universidad Nacional de Colombia, Calle 59 A No 63-020, Autopista Norte, Medellín, AA 3840, Colombia of oxygen only by the striatum mitochondria, and do not include that which takes place in the process of paraquat redox cycling. The authors consider the decreases shown in this Fig. 2a, for paraquat state 4 as well as for paraquat state 3 as strong evidence of a dysfunction of the bioenergetics of the mitochondria isolated from the striatum of the brain upon acute paraquat administration. But these data do not reflect a mitochondrial dysfunction. The RC is the fundamental parameter that indicates the energetic coupling of the mitochondria, and the researchers Czerniczyniec et al. consider that to be the case, writing (see page 400, first paragraph): "The respiratory control ratio (the most sensitive indicator of mitochondrial oxidative phosphorylation coupling)." Nevertheless, these researchers do not report the RC calculated on the basis of their data presented in Fig. 2a (KCN-sensitive respiration). On calculating the $\mathrm{RC}(\mathrm{RC}=$ state $3 /$ state 4$)$ on the basis of these data, (Fig. 2a of the article by Czerniczyniec et al. (2015)), an average value for the control RC of 5.9 and for the paraquat $\mathrm{RC}$ of 11.0 is obtained. These values show something surprising: the paraquat striatum brain mitochondria exhibit a greater degree of oxidative phosphorylation coupling, indicating that they are no subject to a dysfunction of their energetic state upon acute paraquat administration. In the same way, it is curious to observe (in the "apparent" parameters of the mitochondrial respiration shown in Table 1 of the article by Czerniczyniec et al. (2015)) for the RC of the cerebral cortex mitochondria (control $\mathrm{RC}=4.6 \pm 0.2$ and paraquat $\mathrm{RC}=5.3 \pm 0.3$ ) indicate that the cerebral cortex mitochondria isolated from paraquat treated rats exhibit a tendency to be better energetically coupled. The foregoing also justifies the measurement of all of the $\mathrm{KCN}$-sensitive mitochondrial respiration parameters for the cortex brain mitochondria, in which the consumption of oxygen only by the mitochondria is included, and that which takes place in the process of paraquat redox cycling is not. 
Czerniczyniec et al. (2015) consider the decrease of paraquat state 4 as well as paraquat state 3 , due to the negative functional alterations, deducible from Fig. 1 of the article by Czerniczyniec et al. (2015), experienced by mitochondrial complex I and by mitochondrial complex IV. However, negative alterations in the functioning of these mitochondrial complexes have no reason to be present when in fact paraquat striatum brain mitochondria exhibit better oxidative phosphorylation coupling; rather there is a lower quantity of mitochondria in the paraquat treated samples, but with better energetic functioning than the control mitochondria. An analysis of the methodology used in this article reveals possible experimental artefacts that could have given rise to the suggested decrease in the enzymatic activities of mitochondrial complex I-III and of mitochondrial complex IV upon acute paraquat administration.

Czerniczyniec et al. (2015) used submitochondrial membranes for the determination of all of the mitochondrial complex activities, not making reference to the method used for their preparation. But it is very possible that they have been prepared in the same manner as these researchers did in their published study (Czerniczyniec et al. 2011), in which a mixture of fragments of outer and inner mitochondrial membranes was obtained by freezing and thawing the mitochondrial preparation. In the outer membrane of the mitochondria, including the brain mitochondria (Ito 1980), an NADH-cytochrome c reductase system is found, made up of a flavoprotein- 5 and a cytochrome $b_{5}$, facing the cytosol. Lemeshko (2001 and 2002) showed in an exemplary way that in the presence of NADH and cytochrome c (both exogenous), the transport of electrons between the $\mathrm{NADH}-$ cytochrome $\mathrm{c}$ reductase system of the outer mitochondrial membrane (OMM) and complex IV of the inner mitochondrial membrane (IMM) is drastically activated when the OMM is permeabilized or has undergone a rupture (see Fig. 1 in the current Letter to the Editor). The oxidation of the NADH by the

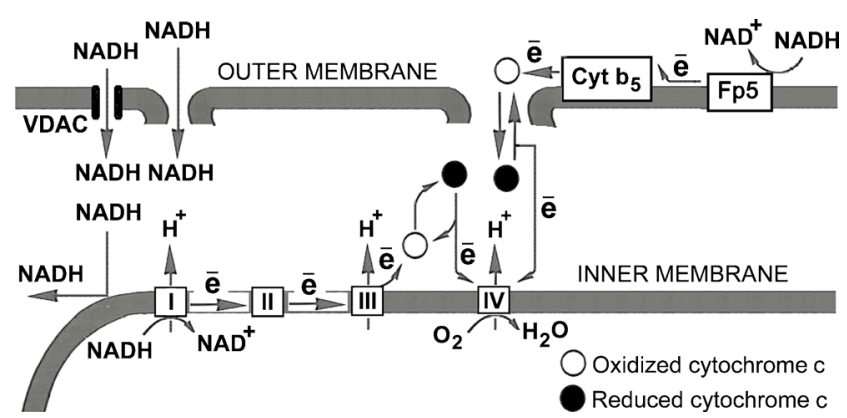

Fig. 1 The external and internal mitochondrial pathway of NADH oxidation. Cyt $b_{5}$ and Fp5 is the cytochrome $b_{5}$ and the flavoprotein-5, respectively. The inner mitochondrial membrane is impermeable for NADH (Lehninger 1978). Cytochrome c passes through the outer mitochondrial membrane only if this membrane becomes permeable for this molecule or if the membrane has experienced a rupture (as is the case shown in this figure)
$\mathrm{NADH}-$ cytochrome $\mathrm{c}$ reductase system of the OMM is called the external mitochondrial pathway of NADH oxidation, different from the internal mitochondrial pathway of NADH oxidation, which consists (see Fig. 1 in the current Letter to the Editor) of the oxidation of the NADH, present in the mitochondrial matrix, by Complex I of the IMM, and the electrons resulting from this oxidation participate in the reduction of different components of the respiratory chain (including the cytochrome c, present in the space between the IMM and the OMM; see Fig. 1 in the current Letter to the Editor). Therefore, under the experimental conditions employed in the study by Czerniczyniec et al. (2015) for the determination of mitochondrial complex I-III activity, the NADH and the cytochrome c, both exogenous, are being oxidized and reduced, respectively, by two different enzymatic systems: by the NADH-cytochrome c reductase system present in the fragments of the OMM and by the mitochondrial complex I-III of the inner mitochondrial membrane fragments, in the case of free access to the electron donor and the electron acceptor. Therefore, the enzymatic mitochondrial activity reported in Fig. 1a of the article by Czerniczyniec et al. (2015) is not an enzymatic activity exclusive to mitochondrial complex I-III. But it is on the basis of the results of Fig. la and b, in Czerniczyniec et al. (2015), that the authors conclude the existence of a dysfunction in mitochondrial complex I of striatum mitochondria during acute paraquat toxicity.

On the other hand, it is possible that during the preparation of the mixture of outer and inner membranes, a fraction of the fragments of the IMM formed vesicles, with the catalytic part of mitochondrial complex I facing their interior. The probability of the formation of these vesicles is high, since when the mitochondria are subjected to a water-lysis the formation of these vesicles is observed (Pedersen et al. 1978), indicating that these mitochondrial inner membrane vesicles are energetically stable. The rate of cytochrome c oxidation by mitochondrial complex IV located in an unclosed membrane fragment is higher than that for one located in a membrane vesicle. This is due to the fact that in the latter case the oxidation of the reduced cytochrome $\mathrm{c}$ is coupled to the generation of an electrochemical potential of $\mathrm{H}^{+}$ ions across the membrane of the vesicle (see Fig. 1 in the current Letter to the Editor), this constituting a limiting factor for the manifestation of the maximum enzymatic activity of the mitochondrial complex IV. One can "visualize" the possible existence of these membrane vesicles in the biological samples, control and/or treated, spectrophotometrically registering the rate of oxidation of reduced cytochrome $\mathrm{c}$ with and without FCCP. In a considerable presence of membrane vesicles, an increase in the slope of the FCCP record of the oxidation of reduced cytochrome $\mathrm{c}$ is to be expected. Now if in the paraquat treated samples, for the striatum brain mitochondria, more membrane vesicles were formed than in the control samples, the decrease in the activity of the mitochondrial complex IV shown in Fig. 1c of the study by Czerniczyniec et al. (2015) is to be expected. In order to discard the aforementioned 
experimental artefact, the best way is to determine the activity of mitochondrial complex IV in the presence of one mitochondrial uncoupler. Thus all of the mitochondrial complexes IV present in the biological samples will be operating at their maximal enzymatic activity. The possible formation of mitochondrial inner membrane vesicles (with the catalytic part of mitochondrial complex I facing their interior) in the biological samples is an additional, and serious, problem in the methodology used by Czerniczyniec et al. (2015) for the determination of the activity of mitochondrial complex I-III. In this case, the exogenous NADH cannot cross the membrane of the vesicle, because the IMM is impermeable for NADH (Lehninger 1978), and so it cannot be oxidized by mitochondrial complex I.

Because of all of what has been described above with respect to the work of researchers Czerniczyniec et al. (2015), it is fundamental to repeat all of the experiments described in this Letter to the Editor, and in this way it will be possible to clarify the true behavior of brain mitochondria upon acute paraquat administration, something that is fundamental in biomedical research due to the negative impact that the use of paraquat has on public health.

\section{References}

Czerniczyniec A, Karadayian AG, Bustamante J, Cutrera RA, LoresArnaiz S (2011) Paraquat induces behavioral changes and cortical and striatal mitochondrial dysfunction. Free Radic Biol Med 51(7): $1428-1436$

Czerniczyniec A, Lanza EM, Karadayian AG, Bustamante J, LoresArnaiz S (2015) Impairment of striatal mitochondrial function by acute paraquat poisoning. J Bioenerg Biomembr 47(5):395-408

Ito A (1980) Cytochrome b5-like hemoprotein of outer mitochondrial membrane: OM cytochrome b. II. Contribution of OM cytochrome $\mathrm{b}$ to rotenone-insensitive NADH-cytochrome $\mathrm{c}$ reductase activity. J Biochem 87(1):73-80

Lehninger AL (1978) Fosforilación oxidativa, estructura mitocondrial y compartimentación del metabolismo respiratorio. In: Lehninger AL, Bioquímica. 2da edición. Ediciones Omega, S.A. pp 519-553

Lemeshko VV (2001) Failure of exogenous NADH and cytochrome $\mathrm{c}$ to support energy-dependent swelling of mitochondria. Arch Biochem Biophys 388(1):60-66

Lemeshko VV (2002) Cytochrome c sorption-desorption effects on the external NADH oxidation by mitochondria: experimental and computational study. J Biol Chem 277(20):17751-17757

Pedersen PL, Greenawalt JW, Reynafarje B, Hullihen J, Decker GL, Soper JW, Bustamante E (1978) Preparation and characterization of mitochondria and submitochondrial particles of rat liver and liver-derived tissues. Methods Cell Biol 20:411-481 